\title{
Monitoring of Anesthesia by Bispectral Analysis of EEG Signals
}

\author{
Lamia Bouafif $\mathbb{C D}$ \\ National Institute of Biomedical Studies of Tunis-Image and Signal Processing Laboratory ENIT, \\ University of Tunis Manar, Tunisia \\ Correspondence should be addressed to Lamia Bouafif; b2lamia@yahoo.fr
}

Received 22 March 2021; Accepted 25 August 2021; Published 21 September 2021

Academic Editor: Michele Migliore

Copyright (c) 2021 Lamia Bouafif. This is an open access article distributed under the Creative Commons Attribution License, which permits unrestricted use, distribution, and reproduction in any medium, provided the original work is properly cited.

Background. In intensive care, monitoring the depth of anesthesia during surgical procedures is a key element in the success of the medical operation and postoperative recovery. However, despite the development of anesthesia thanks to technological and pharmacological advances, its side effects such as underdose or overdose of hypnotics remain a major problem. Observation and monitoring must combine clinical observations (loss of consciousness and reactivity) with tools for real-time measurement of changes in the depth of anesthesia. Methodology. In this work, we will develop a noninvasive method for calculating, monitoring, and controlling the depth of general anesthesia during surgery. The objective is to reduce the effects of pharmacological usage of hypnotics and to ensure better quality recovery. Thanks to the overall activity of sets of neurons in the brain, we have developed a BIS technique based on bispectral analysis of the electroencephalographic signal EEG. Discussion. By collecting the electrical voltages from the brain, we distinguish light sleep from deep sleep according to the values of the BIS indicator (ranging from 0: sleep to 100:wake) and also control it by acting on the dosage of propofol and sevoflurane. We showed that the BIS value must be maintained during the operation and the anesthesia at a value greater than 60. Conclusion. This study showed that the BIS technology led to an optimization of the anesthetic management, the adequacy of the hypnotic dosage, and a better postoperative recovery.

\section{Introduction}

Clinical monitoring is based on the analysis of nervous reactions to stimulation movements such as responsiveness to surgical incision and loss of verbal contact. The consequences of an unsuitable anesthesia are an increase in morbidity, overdosage. (hypotension and respiratory depression), or underdosage (memorization, movement, hypertension, and bronchospasm) [1].

This makes it essential to evaluate the depth of the anesthesia in order to optimize its adequacy to the intensity of the operative stimuli. Historically, the measurement of the depth of anesthesia began with the analysis of the relationship between nociceptive stimuli and the presence or absence of clinical signs such as loss of consciousness, movement, changes in respiratory rate, changes in eye response, and cardiovascular effects. It was in 1875 that an English doctor recorded for the first time the electrical potential of a brain.

In 1929, electroencephalography was invented by a German physician called Hans Berger, in order to study the electrical activity of the brain by measuring the electrical potential. This invention was recognized and completed by the physician Edger Adrian in 1934 [2].

In fact, the use of anesthesia depth monitoring emerged following a recommendation from the American Society of Anesthesiologists (ASA) in 2006, then a Cochrane Library meta-analysis in 2007 and a formal recommendation from SFAR experts in 2009. After 2000, two major studies of Liu's and Punjasawa Dwong's were carried out.

(1) The first one includes 1380 patients from 11 outpatient surgery studies. It found a decrease in hypnotic's consumption of $19 \%$ 


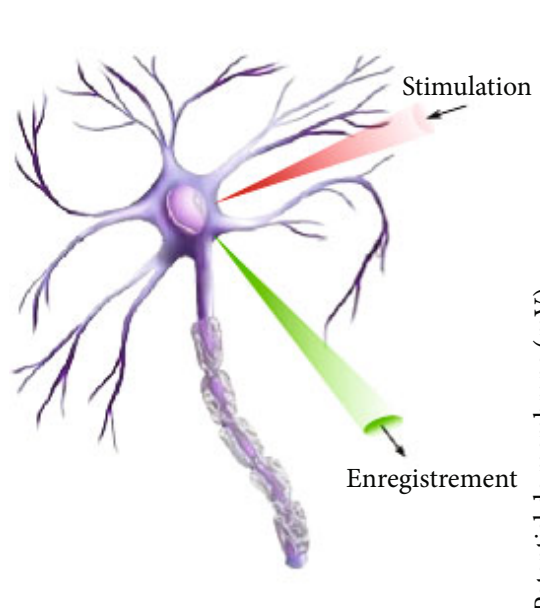

(a)
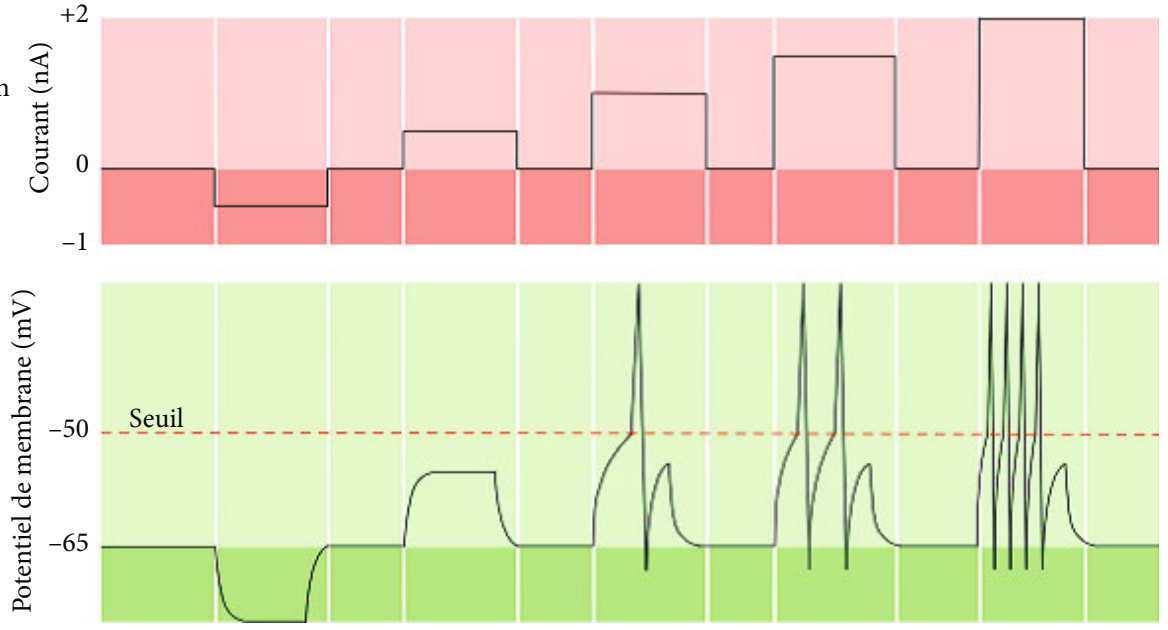

(b) (d) (e)

Figure 1: Principle of the action potential [3].

(2) The second one includes 4056 patients from 20 studies. It found a decrease in propofol consumption of $1.30 \mathrm{mg} / \mathrm{kg} / \mathrm{h}$ and a decrease in halogenated by 0.17 CAM equivalents [3]

Despite the existence of several BIS monitors, several of them suffer from problems such as low signal-to-noise ratio, artifacts, interference with EMG signals, and a medium precision of around $60 \%$ to $80 \%$. In order to surmount these problems, we developed an embedded noninvasive BIS system for monitoring and control the depth of anesthesia during surgery. The first contribution is that we replaced the analog filtering, converters, and electrical circuits with programmable digital filters and embedded algorithms that are implemented in the Raspberry Pi4 electronic board. This avoids inaccuracies of the instrumental circuits and reduces artifact effects. The second contribution is that we succeeded to estimate from the simulation software the optimal dose of hypnotics to achieve a well-determined degree of sleep (for example: BIS $=30 \%$ ), unlike other BIS monitors which adapt the anesthesia boluses with the evolution of BIC values.

1.1. Principle of Electroencephalography EEG. Electroencephalography is a recording of electrical activity in the brain. It represents the trace of temporal variation of electric potential collected on the cranial box for different points of the scalp. The EEG acquisition modality facilitates the visualization of the functioning of the cerebral process and the understanding of neurophysiological phenomena. The acquisition of the EEG signals is carried out from electrodes brought into contact with the scalp.

As EEG is a measure of brain electrical activity, its realization can occur during sleep or in other areas of activity. The received signal is very weak (some microvolts). It varies according to age, sex, the patient state, and alertness.

1.2. Electrical Activity. The brain is a building of a collection of nerve cells called neurons. They are very numerous, around tens of millions. They have the role of circulating nervous messages. Neurons have a long lifespan, and its electrical activity goes through two main phases:

(1) Brain activity to neuron

(2) Surface activity

1.3. Brain-to-Neuron Activity. The electrical activity of neurons depends on the polarization and depolarization of excitable cells. An action potential is a biphasic wave of a few milliseconds, which propagates towards all the nerve cells. It affects human activity such as perception, sleep, and memorization. The information value of the action potential is not transmitted by its amplitude but by its frequency. Therefore, with a depolarization above the threshold, the frequency of the action potential is the highest (Figure 1).

In Figure 1, neuron " A " represents the effect of two microelectrodes placed in the neuron. The first one injects current and the other records the membrane potential. The effect of this stimulation is summarized into 4 portions (B, $\mathrm{C}, \mathrm{D}$, and $\mathrm{E})$. Portion $\mathrm{B}$ represents a result of a negative current, and the neuron is hyperpolarized.

No action potential is produced. Portion $\mathrm{C}$ represents a result of a positive current stimulation, depolarization of the neuronal membrane takes place but it is not sufficient to generate an action potential. For portion $\mathrm{D}$, the injected current depolarizes the membrane to a value greater than the threshold. An action potential is generated. Finally, the discharge frequency of the action potentials will be high with the level of depolarization, proportional to the quantity of current injected; this is the case of portion E.

1.4. Surface Activity. Between two neurons, the interface zone is a surface with a porous membrane which allows the exchange of sodium, chlorine, potassium, and ions. Subsequently, the appearance of an ionic current is observed which propagates towards various cells with a precise frequency. The efficiency of information transfer from one region to another brain level depends mainly on the activity 


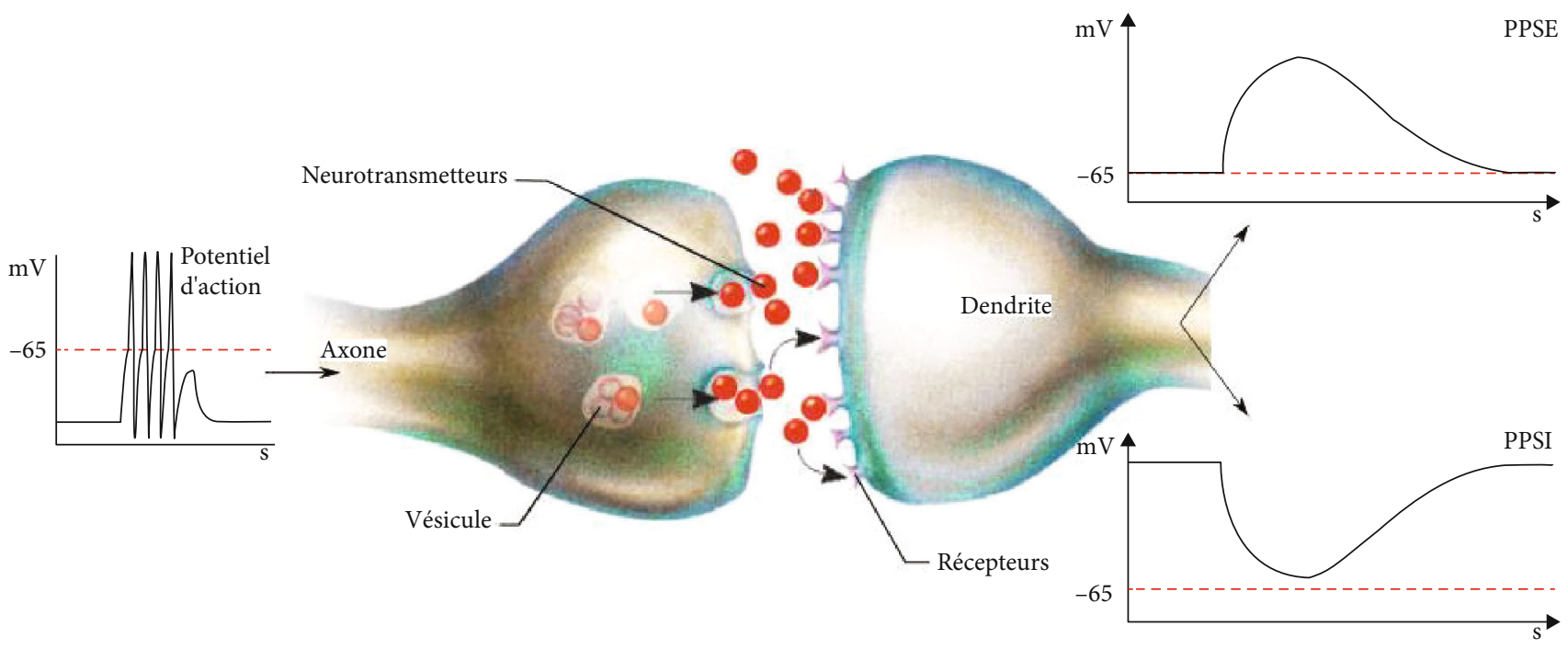

Figure 2: Action potential propagation diagram [2].

level of the cortex. As an action potential propagates along an axon, $\mathrm{Na}+$ ions enter the cell at the active site through voltage-gated $\mathrm{Na}+$ channels. On either side of this depolarization front, a flow of $\mathrm{K}+$ ions emerges via channels open at rest or with delayed opening. As illustrated in Figure 2, this active axonal portion can be assimilated to a current quadruple (two inverted dipoles) [2].

According to the following Figure 3, the polarization of the EEG waves in the surface depends on the position of the synapse in active mode. In fact, the deviation of the potential upwards indicates a negative potential, and conversely, the deviation in the opposite direction indicates a positive potential.

Any EEG electrical signal is characterized by its frequency and its amplitude: the frequencies are between 0.5 to $40 \mathrm{~Hz}$ and the amplitude varies from 5 to 250 microvolts.

In general, amplitudes and frequencies are calculated over a period of $125 \mathrm{~ms}$ to $20 \mathrm{~s}$.

For example, Figure 4 shows an EEG signal recorded by electrodes placed on the scalp surface. We obtained a weak electric field produced mainly by synaptic potentials which can be classified as synchronized waves (c) or irregular: not synchronized (b).

\section{Materials and Methods}

2.1. Description of the Vigilance and Sleep States. Since the development of exploiting electrical activity, the experimental approach to sleep has become scientific. Billiard [5] was the first to identify the state of alertness and sleep during the night. Each state of vigilance corresponds to a specific potential and the use of three parameters (waking state, light and slow sleep state, and slow and deep sleep state). The signal processing of the EEG signal identified five cerebral rhythms: Delta, Theta, Alpha, Beta, and Gamma. These rhythms are classified according to their frequency band and their amplitude according to Table 1.

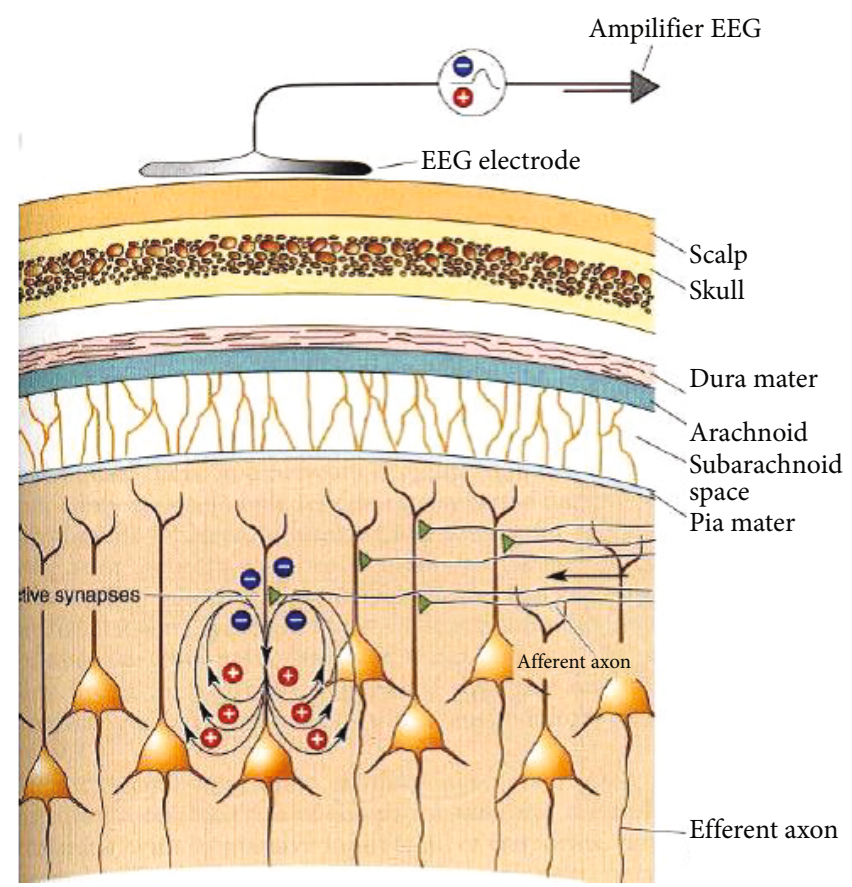

FIGURE 3: The principle of EEG registration [3].

The temporal waveforms of brain waves are illustrated in Figure 5.

2.1.1. Standby State. It is the stage which characterizes the discontinuous emission of alpha wave. We therefore observe a reduction in amplitude which results in the alpha wave flattening.

There are two standby states:

(1) The first is active awakening characterized by the appearance of low amplitudes between 5 and 20 microvolts with a rapid frequency. We are talking about beta wave 


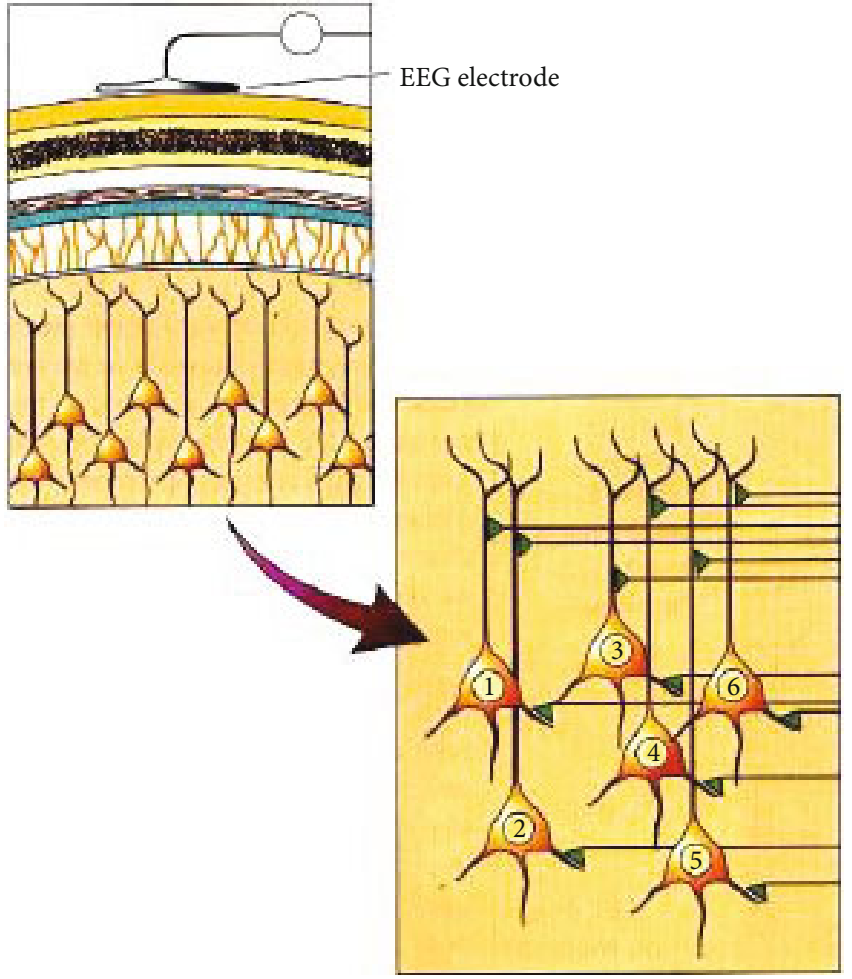

(a)

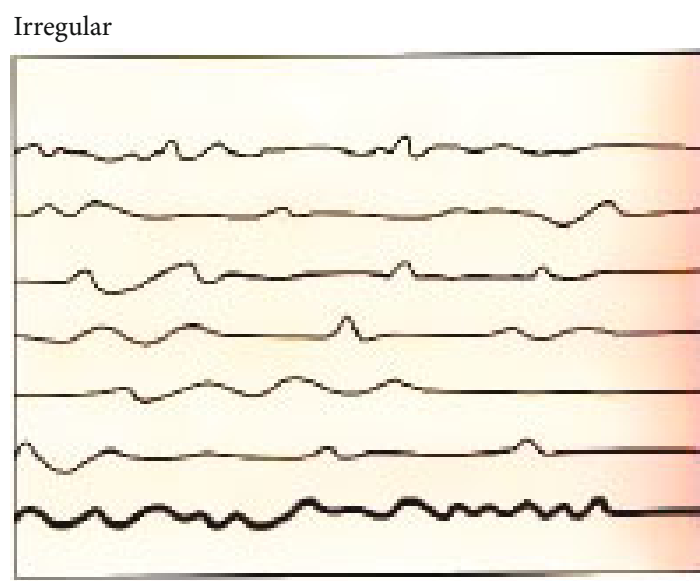

(b)

Synchronized

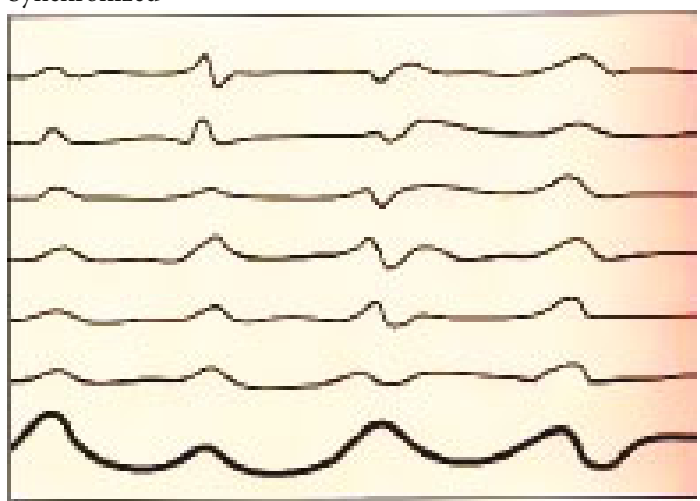

(c)

FIGURE 4: EEG signals related to synaptic activity [4].

TABLE 1: Characteristics of brain waves.

\begin{tabular}{|c|c|c|c|}
\hline Wave & Physio/psycho & Frequency $(\mathrm{Hz})$ & Amplitude $(\mu \mathrm{V})$ \\
\hline Delta & $\begin{array}{c}\text {-Deep sleep } \\
\text { Eye movement: abnormal }\end{array}$ & $0-4$ & 300 \\
\hline Theta & $\begin{array}{l}\text {-Light sleep } \\
\text {-Eyes closed }\end{array}$ & $4-8$ & $50-100$ \\
\hline Alpha & $\begin{array}{l}\text {-Mental calm } \\
\text { Receptivity, relaxation } \\
\text {-Eyes closed }\end{array}$ & $8-12$ & $30-50$ \\
\hline Beta & $\begin{array}{c}\text {-Paradoxical sleep } \\
\text {-Rational thinking } \\
\text {-Eyes open } \\
\text {-Vigilance }\end{array}$ & $12-30$ & 10 \\
\hline Gamma & $\begin{array}{c}\text {-Simultaneous information from different sectors } \\
\text {-Problem resolution }\end{array}$ & $>30$ & - \\
\hline
\end{tabular}




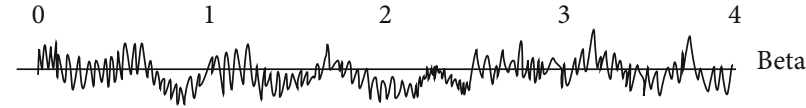

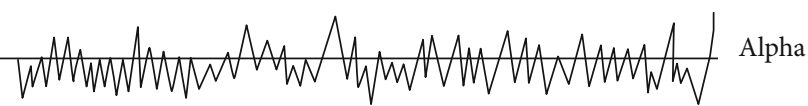
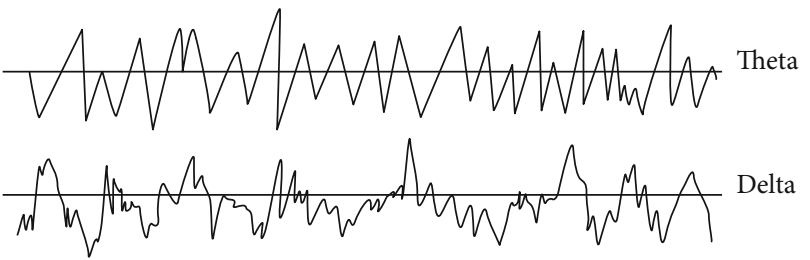

FIGURE 5: Illustration of brain waves [6].

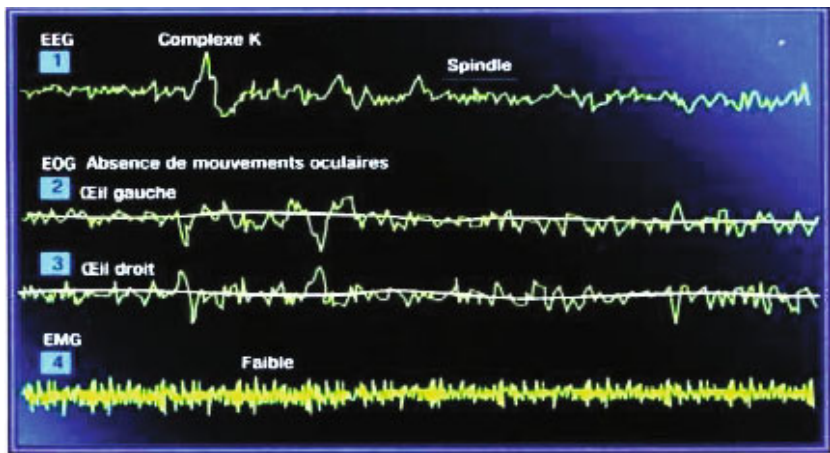

Figure 6: EEG waveforms of the slow and light sleep stage [7]. (1): EEG signal, (2): left eye, (3) right eye, (4) EMG.

(2) However, the second state is calm awakening which corresponds to the alpha wave

2.1.2. Slow and Light Sleep State (Figure 6). It is characterized by a slowing of brain waves with an increase in amplitude, and it breaks down into four stages of increasing depth:

(i) Stage 1. Is a stage between wakefulness and sleep corresponding to theta wave: $10 \%$ of sleep in young adults

(ii) Stage 2. Is a confirmed sleep exhibiting the characteristics of a slow theta wave by breaking down into two types of waves. The first is a sleep spindle wave at a fast (12 to $16 \mathrm{~Hz}$ ) and short-term (1 to $2 \mathrm{~s}$ ) frequency. The second is characterized by the slow wave and appears in a transient and cyclical way

(iii) Stage 3 and 4. The two stages are deep sleep. It presents the characteristic of slow wave delta with higher amplitude than 75 microvolts and a frequency range between 0.5 and $4 \mathrm{~Hz}$

2.1.3. Slow and Deep Sleep State (Figure 7). An essential stage in children because it is the period of secretion of growth hormone which activates all the synthesis processes from the early morning, in particular the protein. The paradoxical slow sleep presents an active center of the hypnogram. It is

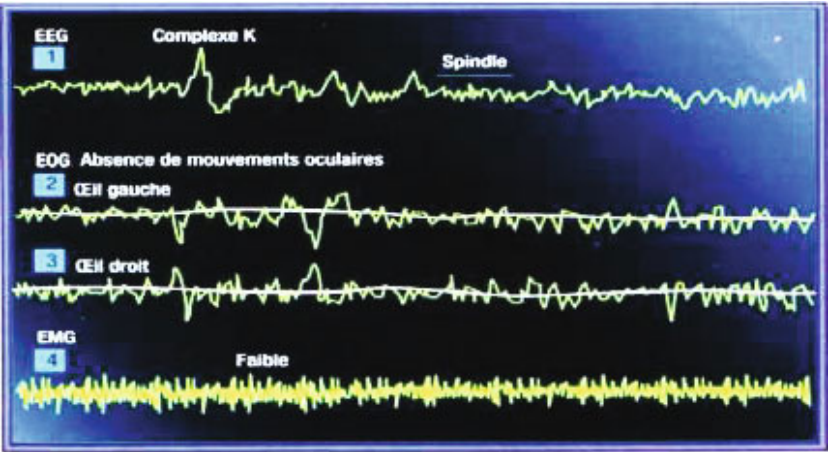

Figure 7: Profile of the GET step of slow and deep sleep [7].

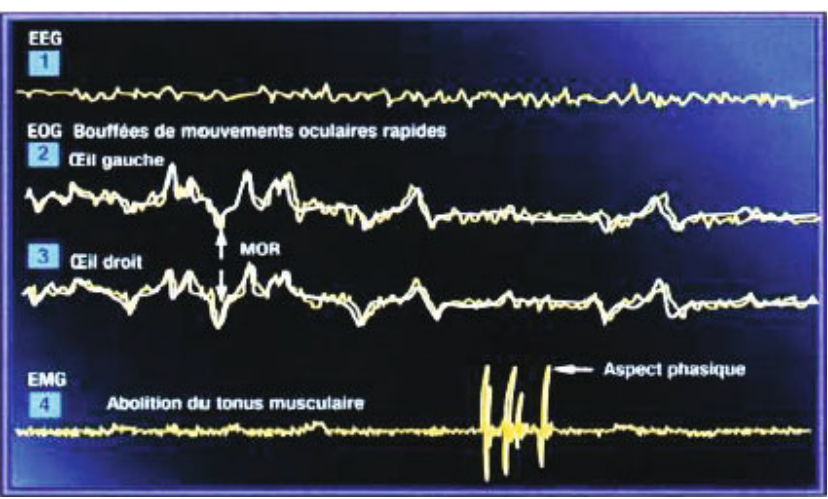

Figure 8: Profile GET step of REM sleep [7].

with the paradoxical sleep that one obtains the expression of the regenerative functions of dream and sleep.

2.1.4. REM Sleep State (Figure 8). This stage of sleep is slow and paradoxical (also called PMO stage, eye movement phase, or REM). This state is shorter with an intensive brain activity. This period is accompanied by rapid eye movement caused by brain waves, which is related to dreams. This hard sleep is between 15 to 20 minutes. It is characterized by low amplitude and rapid pace. It appears for a movement ordered but not realized and louse thought lively, strange, and illogical [8].

2.2. Registration Procedure by Electrodes. For EEG signal acquisition, the sensors are electrodes which record the variations in electrical potential which are a few millimeters in radius. The electrodes are inserted in an elastic cap. The bonnet that is placed on the head of a patient is decomposed of a tissue and electrodes. The electrodes are of the "GrayWalter" type which consists of a silver rod covered with a tissue plug inhibited by a saline solution. They are fixed in a stabilizing support which allows them to stand upright on the scalp. They are held by a small hook which is a net made in the crisscrossed rubber strap that is fixed on the subject's head in order to keep the electrodes in suitable places. As illustrated in Figure 9, each letter indicated on the electrodes a specified region. Even numbers indicate the right side and the odd numbers indicate the left dimension. 


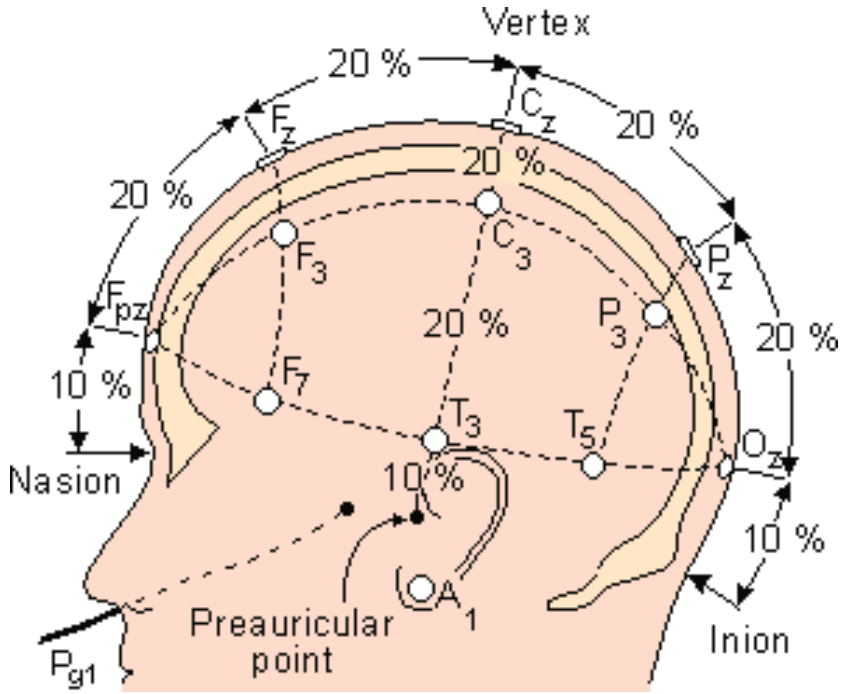

(a)

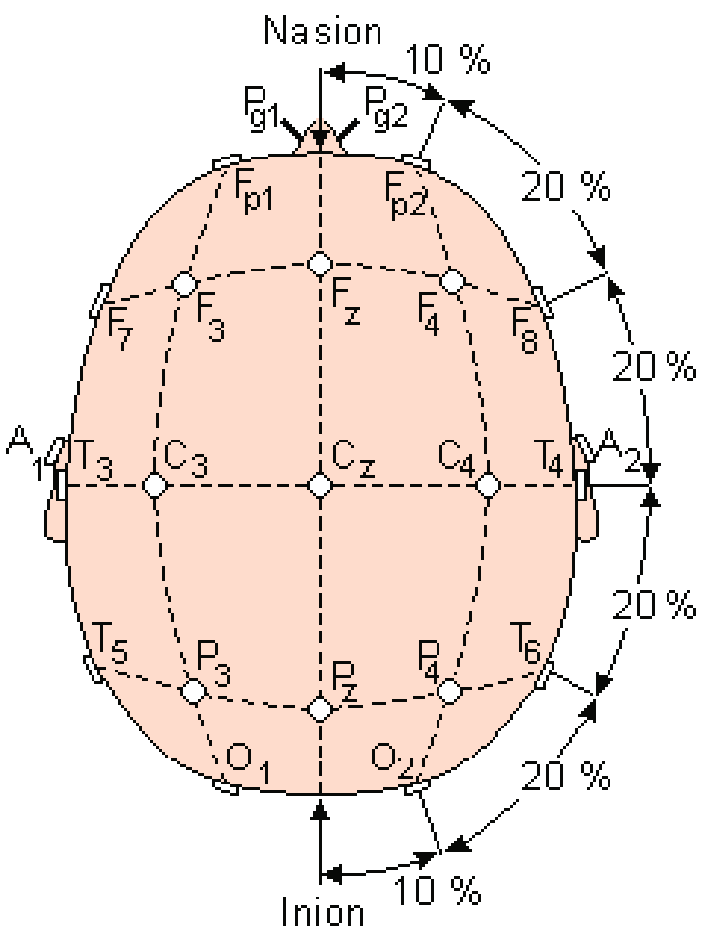

(b)

Figure 9: Localization of EEG electrodes [7]. F: frontal; Fp: polar front; A: earlobe; C: central; O: occipital; T: temporal; Z: central axis; P: parietal.

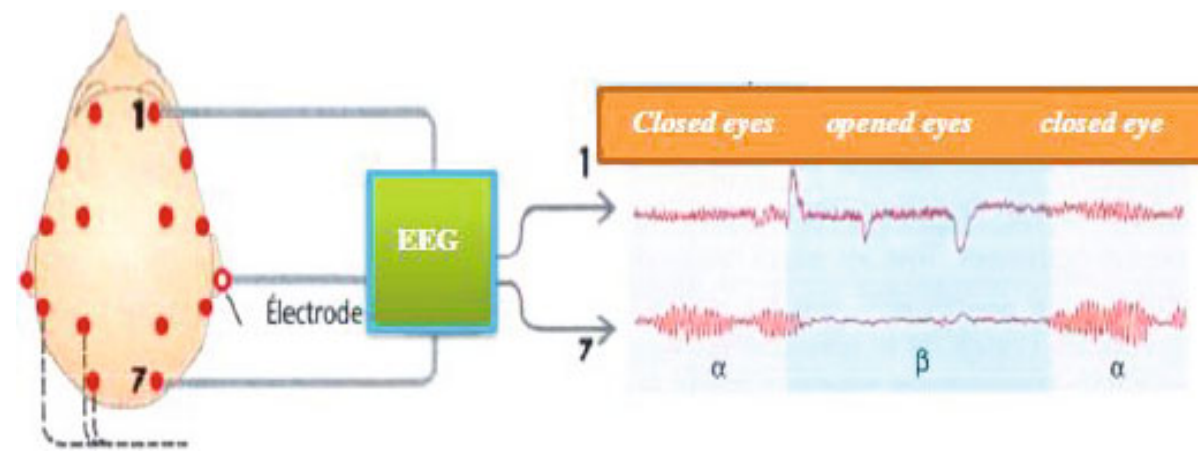

Figure 10: EEG measuring system during eye movements [6] (closed, opened).

2.2.1. Rhythmic Activity. The form and intensity of EEG electrical activity depend on the position of the recording electrodes and on brain activity (Figure 10). They depend on the location of the electrodes, their impedances, and the state of sleep. Electroencephalography (EEG) is used to record the rhythmic activities of the cerebral cortex, classified into five waves: Delta, Theta, Alpha, Beta, and Gamma [9].

2.3. The BIS Procedure. The study of the energy spectrum of cortical signals shows the existence of frequency bands that are related to behavioral observations. The frequency waves are divided as follows [10]:

(1) Delta band (0 to $4 \mathrm{~Hz}$ )

(2) Theta band (4 to $8 \mathrm{~Hz}$ )
(3) Alpha band (8 to $12 \mathrm{~Hz}$ )

(4) Beta band (12 to $25 \mathrm{~Hz}$ )

(5) Gamma band $(>30 \mathrm{~Hz})$

Power spectral analysis gives information on amplitude and frequency but does not take into account the phase between the components of different frequencies. The firstorder statistics lose all phase information. In order to retrieve the statistical information, we introduce the bispectral analysis [11].

\subsection{Bispectral Analysis}

2.4.1. Quadratic Phase Coupling (QPC). To be able to quantify the quadratic phase coupling between pairs of frequencies, it is necessary to calculate from bispectrum. The 


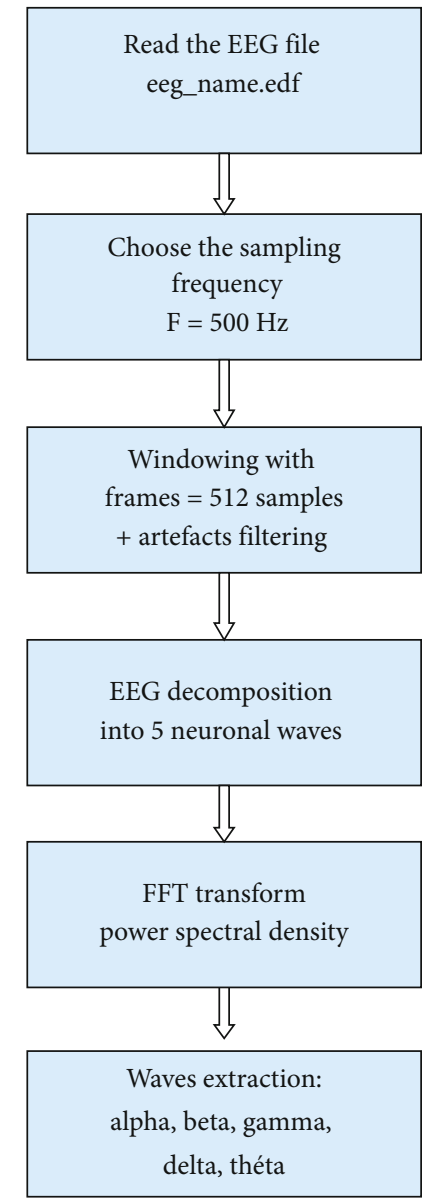

FIgURE 11: Wave extraction algorithm.

interaction of two wave trains of frequencies $f 1$ and $f 2$ can generate two interaction waves of frequencies $\left(f_{1}+f_{2}\right)$ and $\left(f_{1}-f_{2}\right)$.

The frequency components can therefore interact and produce other mixed frequency components whose wave numbers and frequencies are formed from the sum or the difference of these primary components. The frequencies $f_{1}, f_{2}$, and $f_{3}$ can be related by a quadratic nonlinear interaction if the following equation is satisfied [12]:

$$
f_{1} \pm f_{2} \pm f_{3}=0 .
$$

In general, if we have a signal which is composed of three sinusoids with frequencies and phases $(\omega 1, \phi 1)$, $(\omega 2, \phi 2)$, and $(\omega 3, \phi 3)$, the sinusoids 1 and 2 are said to be quadratically coupled in phase (QPC) if and only if [13]:

$$
\begin{gathered}
\omega 1 \pm \omega 2 \pm \omega 3=0, \\
\varphi 1 \pm \varphi 2 \pm \varphi 3=0
\end{gathered}
$$

2.5. Bispectrum and Bicoherence. The bispectrum is a complex quantity that measures nonlinear interactions in a process that generates a signal and the correlation between the phases of a signal at different Fourier frequencies. So the analysis bispectral is defined as a FFT-2D, and bispectrum is the Fourier transform of the sequence of third order cumulate of a random process.

Despite its capacity for calculation, the bispectrum remains complex to understand it. It is for this reason one calls upon the bicoherence [11]. This index varies between $0 \%$ and $100 \%$.

2.5.1. Calculation of the Bispectrum. To compute the bispectrum, EEG signals are first divided into a series of epochs. Then, Fourier transform $X_{j}(f)$ of each epoch is computed. The bispectrum $B\left(f_{1}, f_{2}\right)$ is calculated from the following equations:

$$
\begin{aligned}
\operatorname{TP}_{j}\left(f_{1}, f_{2}\right) & =X_{j}\left(f_{1}\right) \cdot X_{j}\left(f_{2}\right) \cdot X_{j}^{*}\left(f_{1}+f_{2}\right), \\
B\left(f_{1}, f_{2}\right) & =\left|\sum_{j} \operatorname{TP} j(f 1, f 2)\right|,
\end{aligned}
$$

where $\mathrm{TP}_{j}$ is the spectral triple product. $X\left(f_{1}\right), X\left(f_{2}\right)$, and $X\left(f_{1}+f_{2}\right)$ are complex values calculated from Fourier transform.

$X *(f)$ is the conjugate of $X(f)$.

For real processes, there are 12 areas of symmetry in the bispectrum [14]. The bicoherence BIC is defined as the degree of standardized BIS (between 0\% and 100\%). The normalized value of the bispectrum is called bicoherence $\operatorname{BIC}\left(f_{1}, f_{2}\right)$. It is calculated from the following equation [15]:

$$
\operatorname{BIC}\left(f_{1}, f_{2}\right)=\frac{B\left(f_{1}, f_{2}\right)}{\sum_{j}\left|\operatorname{TP} j\left(f_{1}, f_{2}\right)\right|} \cdot 100,
$$

The numerator is different from the denominator because:

$$
\left|\sum \mathrm{TP}_{j}\right| \leq \sum|\mathrm{TP} j|
$$

This expression can be expressed in function of the power spectral density $P(f)$ as:

$$
\operatorname{BIC}\left(f_{1}, f_{2}\right)=\frac{B\left(f_{1}, f_{2}\right)}{\sum_{j} \sqrt{P j\left(f_{1}\right) \cdot \operatorname{Pj}\left(f_{2}\right) \cdot \operatorname{Pj}\left(f_{1}+f_{2}\right)}} \cdot 100 .
$$

The last formula can be deduced as:

$$
\begin{aligned}
\left|\mathrm{TP}_{j}\left(f_{1}, f_{2}\right)\right|^{2} & =\left.\left|X_{j}\left(f_{1}\right)\right|^{2} \cdot X_{j}\left(f_{2}\right)\right|^{2} \cdot\left|X_{j}^{*}\left(f_{1}+f_{2}\right)\right|^{2} \\
& =P_{j}\left(f_{1}\right) \cdot P_{j}\left(f_{2}\right) \cdot P_{j}\left(f_{1}+f_{2}\right) .
\end{aligned}
$$

Then

$$
\left|\mathrm{TP}_{j}\left(f_{1}, f_{2}\right)\right|=\sqrt{P_{1}\left(f_{1}\right) \cdot P_{j}\left(f_{2}\right) \cdot P j\left(f_{1}+f_{2}\right)} .
$$

With the power spectral density $P_{j}(f)=\left|X_{j}(f)\right|^{2}$. 


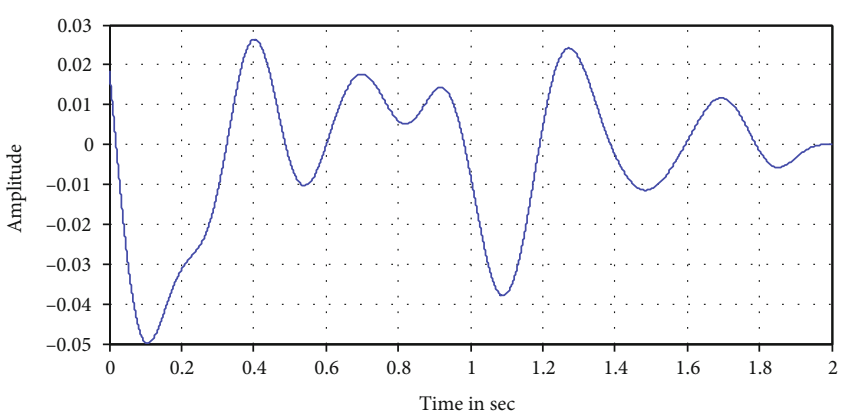

Figure 12: The delta wave: deep sleep.

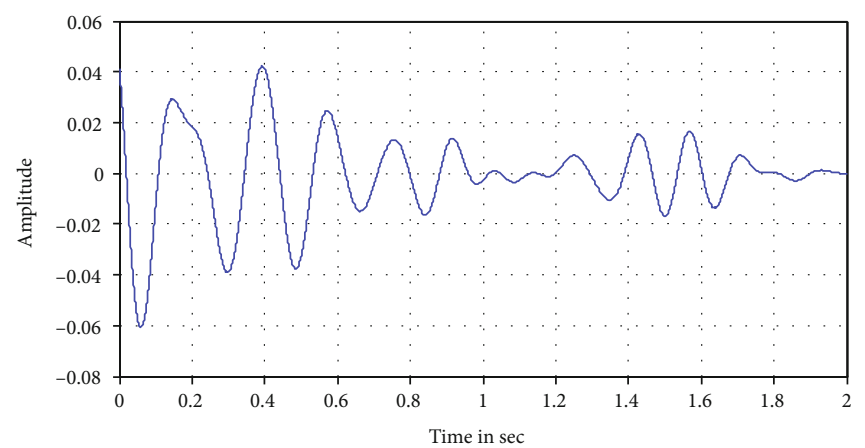

Figure 13: The theta wave: light sleep.

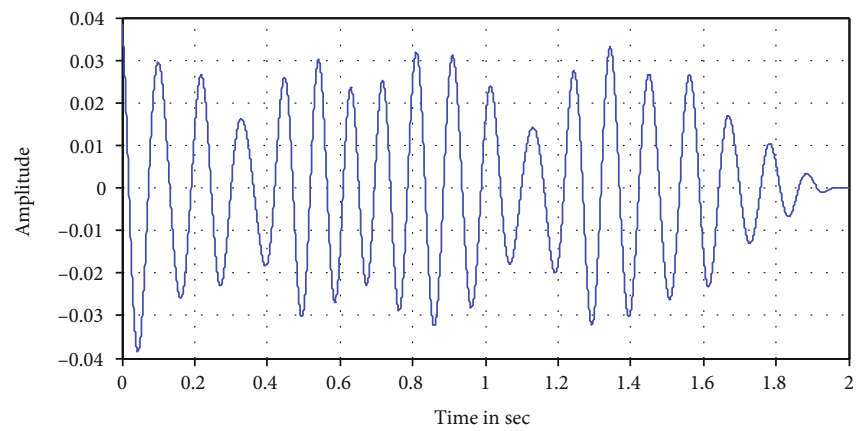

Figure 14: Alpha wave: brain sleeping

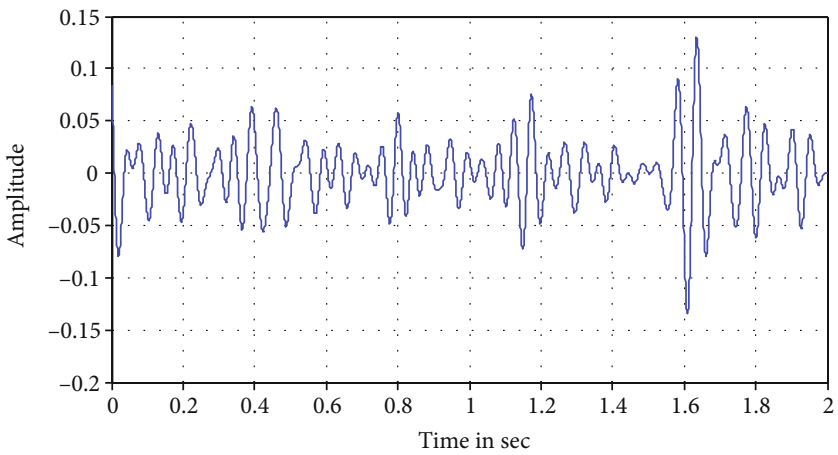

Figure 15: Beta wave: first activity/eye's opening. 


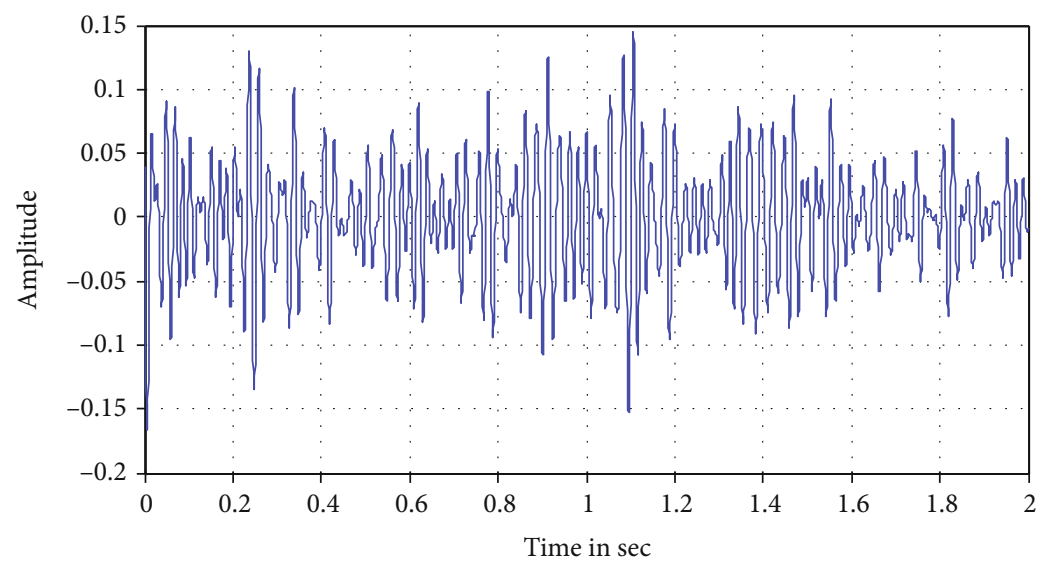

FIgURE 16: The gamma wave: information processing.

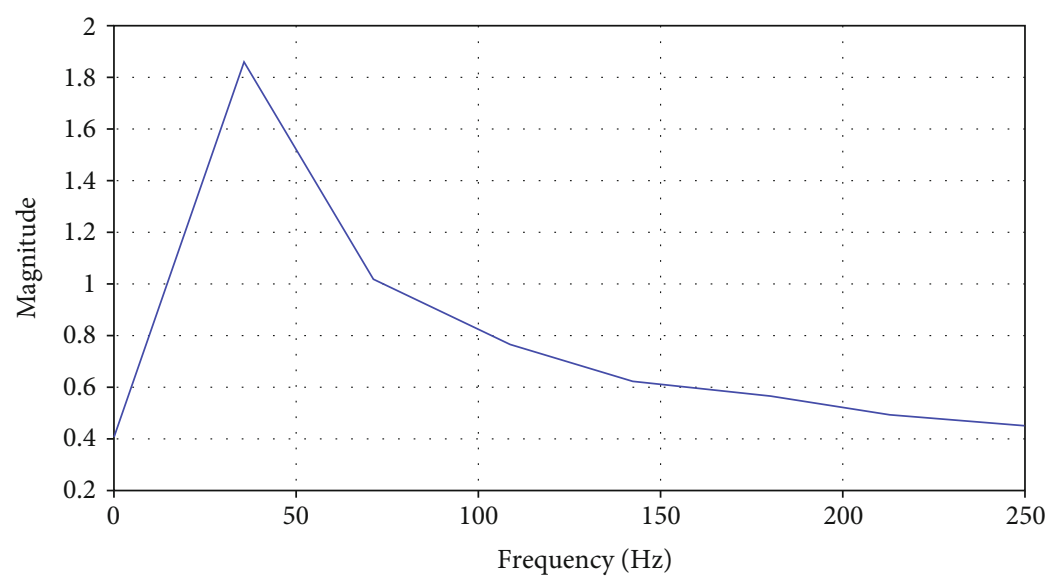

Figure 17: Spectral density of an extract of the EEG signal.

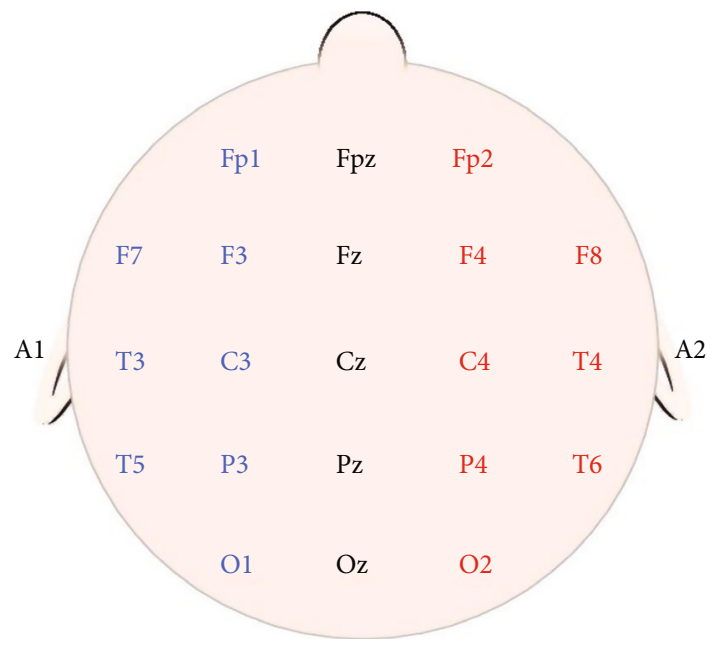

FIGURE 18: Electrodes positions.

\section{Results and Discussion}

Figure 11 illustrates the principle of the EEG segmentation and wave's discrimination. We start by reading the data from the EEG recordings which are saved in a called "file_ name.edf".

Then, the EEG signal is into frames of 512 points with a sampling frequency of $500 \mathrm{~Hz}$. We thus obtain the cerebral rhythms (Alpha, Beta, Theta, Delta, and Gamma). Finally, we apply the fast Fourier transform (FFT) in order to separate and to identify the 5 waves: Delta $(0-4 \mathrm{~Hz})$, Theta $(4$ to $8 \mathrm{~Hz})$, Alpha $(8-12 \mathrm{~Hz})$, Beta $(12$ to $30 \mathrm{~Hz})$, and Gamma $(>30 \mathrm{~Hz})$.

3.1. Simulation. The following Figures $12-16$ represent the results of the spectral analysis of the EEG signals, called the brain waves. Every one of these five signals represents a specific state of the patient.

Figure 17 shows the spectral density of the EEG signal. The beta wave portion is characterized by a peak, observed at $30 \mathrm{~Hz}$. The beta brain wave appears in a patient with open eyes and for a frequency of 12 to $30 \mathrm{~Hz}$. The obtained results show that from calculation of the spectral density of the signal, it is possible to identify the patient case where it is not always sufficient. 


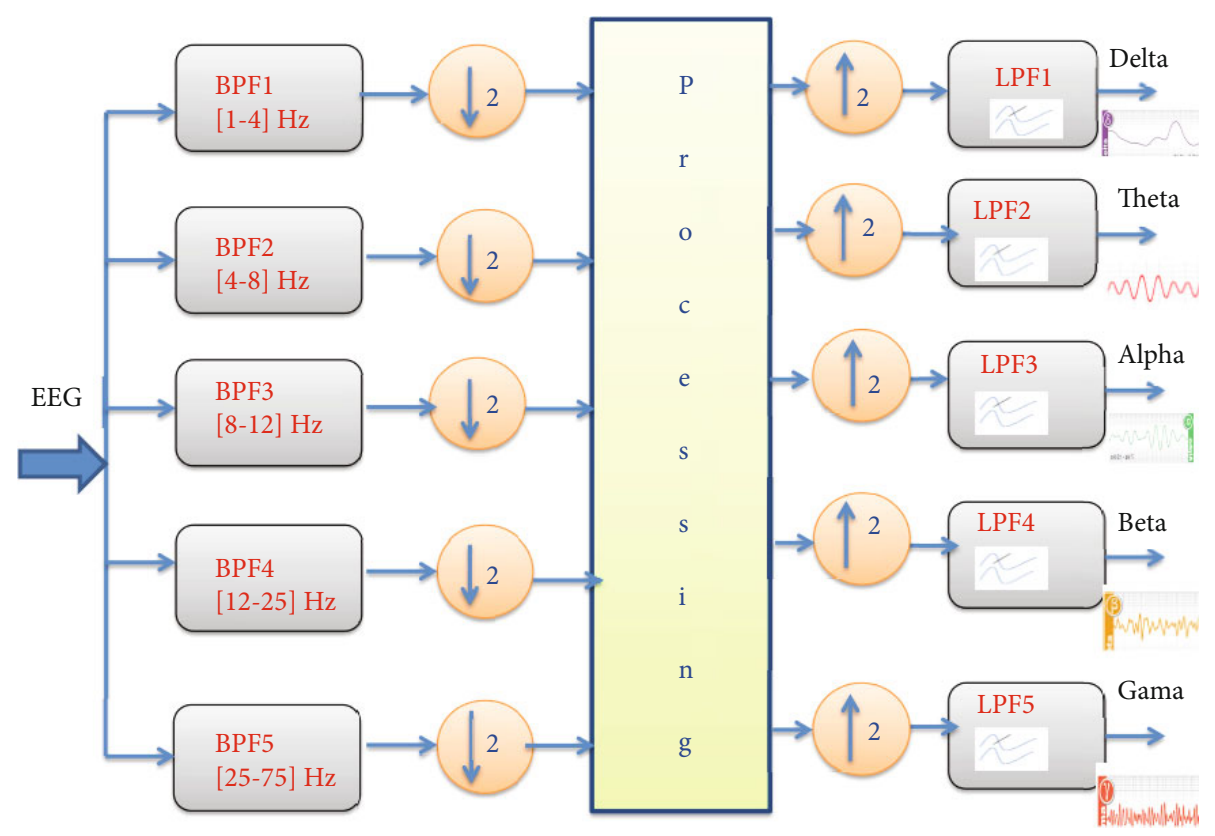

FIgURE 19: Filterbank decomposition of EEG signals.

3.2. Database and Experiment Protocols. In section, we will study two different signals in order to detect quadratic phase coupling:

(i) The first signal processed is an EEG examination result for a healthy patient (male, 25 years old: not affected by neurological diseases such as epilepsy), calm with open eyes

(ii) The second signal processed from a patient (26-yearold woman) under anesthesia (with $2.6 \%$ sevoflurane)

The protocol for the measurements is as follows:

(i) EEG signals are collected from electrodes placed at defined locations on the scalp. The placement of the electrodes is defined according to Figure 9

(ii) We used the 10-20 system because it identifies the same relative position on the scalp regardless of head size. It is based on meridians crossing the scalp into landmarks such as nasion, inion, left, and right auditory tragus

The "10-20" refers to the fact that actual distances between adjacent electrodes are either $10 \%$ or $20 \%$ of the total front-back or right-left distance of the skull (Figure 9). Each electrode placement site has a letter to identify the lobe, or area of the brain: the standard positions and areas are classified as illustrated in Figure 18: prefrontal (Fp), frontal $(\mathrm{F})$, temporal $(\mathrm{T})$, parietal $(\mathrm{P})$, occipital $(\mathrm{O})$, and central (C). There are also $(Z)$ sites for electrodes placed on the midline sagittal plane of the skull, $(\mathrm{Fz}, \mathrm{Cz}, \mathrm{Pz}$, and $\mathrm{Oz})$ which are present mostly for reference-measurement points. Evennumbered electrodes refer to electrode placement on the right side of the head, whereas odd number electrodes refer to the left side.

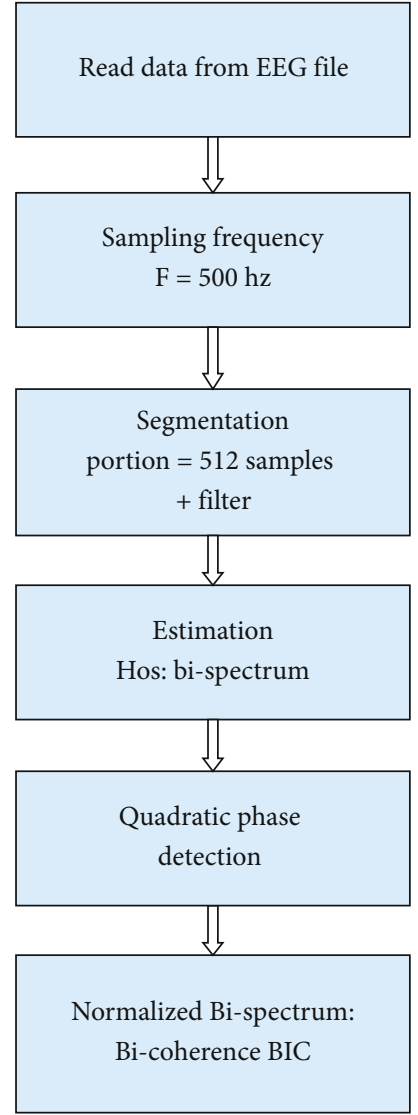

FIGURE 20: BIS and BIC algorithm of an EEG signal. 


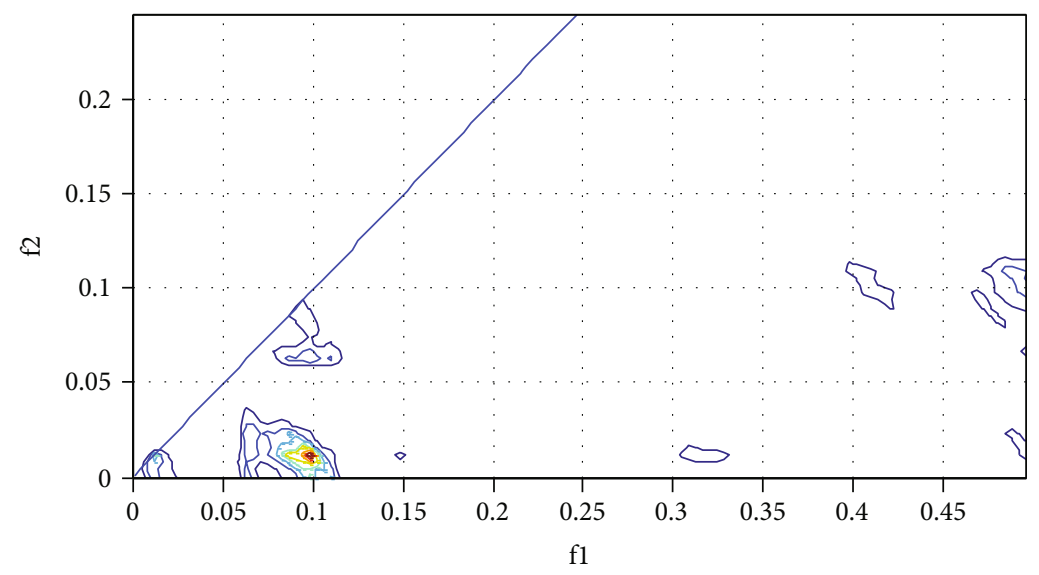

Figure 21: Bispectrum with open eyes.

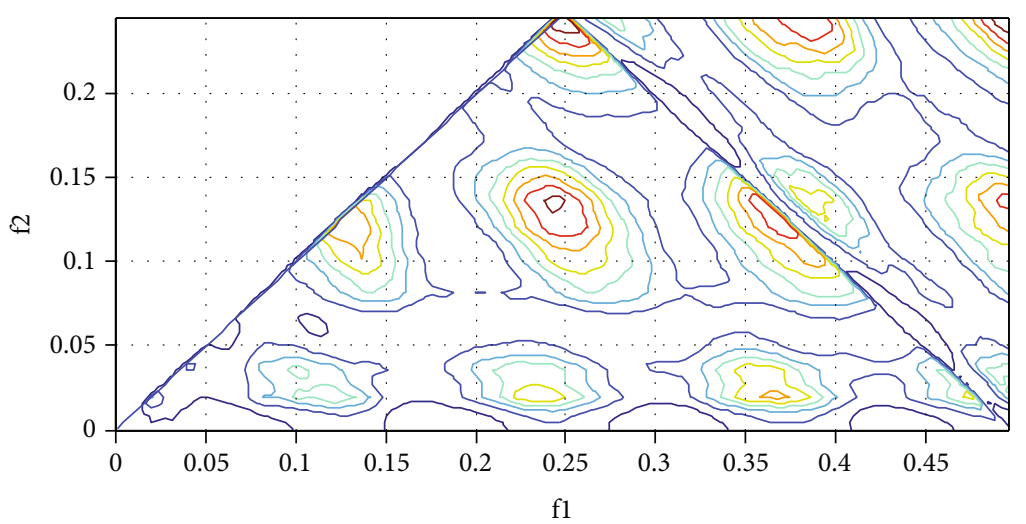

FigURE 22: Bispectrum of a patient under anesthesia (2.6\% sevoflurane).

TABLE 2: Comparison table between the value of BIS and the value found.

\begin{tabular}{lcc}
\hline Patient state & $\begin{array}{c}\text { Theoretical BIS value in } \\
\%\end{array}$ & $\begin{array}{c}\text { Real value in } \\
\%\end{array}$ \\
\hline $\begin{array}{l}\text { Patient under awake } \\
\text { state }\end{array}$ & 100 & 92.2 \\
$\begin{array}{l}\text { Patient under } \\
\text { anesthesia }\end{array}$ & 0 & 3.1 \\
\hline
\end{tabular}

TABLE 3: Identification rate table between the two patient cases.

\begin{tabular}{lcc}
\hline Patient condition & Awake & Under anesthesia \\
\hline Identification ratio (\%) & 90.4 & 96.6 \\
\hline
\end{tabular}

(i) Electrode-gels are used. They act as a malleable extension of the electrode, so that the movement of the cables is less likely to produce artifacts. The gel maximizes skin contact and allows for a lowresistance recording through the skin

(ii) To eliminate the offsets, the voltage is defined as the potential difference between 2 electrodes, grouped by 2. Among the 20 sensors, four electrodes were selected: Fp1-Fp2 and T3-T4
This choice is justified by the ease of access to contact points and the symmetry with respect to the middle head.

The impedance of each electrode is in the order of 1000 ohms.

(i) For signal amplification, we used differential amplifiers. They magnify the difference between two channels or electrodes. The advantage is that, an unwanted signal which is common to the two inputs will be subtracted

(ii) Many signals present on the scalp, include power line interference at $50 \mathrm{~Hz}$ and the electromyogram EMG, which may extend above $100 \mathrm{~Hz}$. To prevent aliasing distortion of the EEG signal, we adopt a sample rate above $250 \mathrm{~Hz}$ ( $\mathrm{Fs}=500 \mathrm{~Hz}$ in our case). The filtering is based on a second-order band pass I.I.R digital filter $[1 \mathrm{~Hz} ; 75 \mathrm{~Hz}]$ to respond to the Nyquist frequency $\mathrm{Fn}=\mathrm{Fs} / 2(\mathrm{Fn}=250 \mathrm{~Hz})$ and to restore alpha, beta waves, gamma, delta waves, and also to eliminate artifacts and network harmonics (Figure 19).

The filter bank is based on a decomposition into five IIR bandpass filters (BPF) related to the EEG waves (delta, theta, alpha, beta, and gamma) followed by a downloading 
TABLE 4: Comparison with similar BIS studies.

\begin{tabular}{|c|c|c|c|c|}
\hline Reference study & Average propofol dose (mg/kg/hour) & Time to consciousness (minutes) & Average number of boluses & Mean error \\
\hline Inaba et al. [19] & 5.3 & 5.7 & 2.3 & 0.2 \\
\hline Weatherburn et al. [20] & 18.4 & 14.6 & - & 0.67 \\
\hline Zhao et al. [21] & 0.95 & 15 & - & 0.09 \\
\hline Lamia (our study) & 2.6 & 3.2 & 1 & 0.03 \\
\hline
\end{tabular}

step by a factor 2 . The five output signals are applied to a preprocessing step constituted by a deconvolution of artifacts/EEG and EMG/EEG by using the cepstral method. Finally, we can restore the enhanced EEG signals by lowpass filters (LPF).

3.3. Algorithm of the BIS Application on the EEG Signal. After the first step of reading data from EEG, the output signals are saved as “*.edf” file. Then, the EEG file is segmented into 512 points per frame with a sampling frequency of $500 \mathrm{~Hz}$. The estimation of HOS is computed by the bispectrum analysis in order to detect the phase of quadratic coupling between the various components of EEG signal. Finally, the normalization of the bicoherence of the bispectrum result was calculated, between 0 and $100 \%$.

This algorithm is similar to PLE (phase lag entropy) that uses four-channel EEG to measure the temporal pattern diversity in the phase relationship of frequency brain signals $[16,17]$.

Figure 20 illustrates the principle of the BIC algorithm. We begin with the analysis of the EEG signal of the patient with open eyes by reading the signal with a sampling frequency Fs $=500 \mathrm{~Hz}$ and a frame length $=512$ samples. Note that when we calculated the spectral power of an awake subject, it is found a peak of $30 \mathrm{~Hz}$ corresponding to the beta brain rhythms. Our aim in this section is to count the quadratic phase coupling QPC. This function detects the quadratically coupled harmonics in phase using the TOR method (third-order recursion) and calculates the bispectrum value of the coupled components. The following figure shows the EEG signal bispectrum of an awake breast subject.

3.4. Simulation Results. The parametric estimation of the bispectrum shows the presence of weak phase coupling (blue outline in Figure 21) between the different components of the EEG signal of the awake patient with a maximum of bispectrum located at the point $\mathrm{B}(0.0976,0.0117)$. The bispectrum result should be normalized across the bicoherence or bispectrum normalized through the BIC equation: $\mathrm{BIC}=92.2 \%$.

Subsequently, we are interested in analyzing the EEG signal of the patient under anesthesia (2.6\% sevoflurane [18]) by reading the signal which is normalized at the same conditions. Note also when we calculated the spectral power of subjects under anesthesia, we found a peak of $2 \mathrm{~Hz}$ corresponds to the cerebral rhythm delta. The following Figure 21 shows the bispectrum of the EEG signal of a patient under anesthesia (2.6\% sevoflurane).
The parametric estimation of the bispectrum shows the presence of a strong phase coupling (presence of red outline in Figure 22) between the different components of the EEG signal of the awake patient with maximum of bispectrum, located at B $(0.49609,0.24609)$, and the presence of other value is given by $\mathrm{B}(0.1312,0.1351),(0.2385,0.1487), \mathrm{B}$ $(0.2502,02460)$. The bicoherence or normalized bispectrum is $\mathrm{BIC}=3.1 \%$. We notice in last Figures 21 and 22 the absence of quadratic coupling for the awake breast patient with a normalized value of $\mathrm{BIC}=92.2 \%$ (Figure 21) and a strong presence of coupling in the patient under anesthesia of a value of $\mathrm{BIC}=3.1 \%$ (Figure 22 ).

Table 2 summarizes the obtained values and results.

The identification ratio of our tests is summarized in the following Table 3.

We can interpret that going towards the value $100 \%$ the marked subject awake with an absence of phase coupling and going towards $0 \%$ the subject marked under anesthesia with a strong presence of coupling. The absence of coupling computed by the bispectrum signifies patient awareness, and the strong presence of coupling signifies the vigilance of the patient. Finally, with our strategy, we obtained an accuracy of $92 \%$ which is very promising and interesting, one compared with other industrial BIS or entropy monitors.

3.5. Comparison with Other Studies. In Table 4, we compared our results with three similar bibliographic studies using as judgment parameters, the sedation-awakening period, the percentage of morphine or sevoflurane, the BIC error, and the number of doses taken during anesthesia. We notice that we obtained similar results to Zhao's study with a decrease in error and consciousness recovery time.

\section{Conclusion}

The study of EEG signals is very complex because it requires the knowledge of biological brain signals and the technologies relating to its components. The aim of this study is to develop a noninvasive method for computing and monitoring in real time the degree of general anesthesia by applying the bispectral analysis of the EEG signal. We developed a methodology of EEG signal segmentation and bispectral analysis in order to extract the five brain waves.

We demonstrated that every wave corresponds to a specific state of the patient. In order to identify the patient state, during and after anesthesia, we computed the synchronization between the components of the spectrum by using the quadratic phase coupling (QPC) strategy. The obtained values of the bispectrum and bicoherence index allowed us to classify and then to recognize the patient state and the 
anesthesia evolution. These results are very interesting because they can assist medical staff to better control and monitoring the anesthesia during surgery operations, reduce the use of hypnotics, and contribute to a better postoperative recovery.

\section{Abbreviations}

BIS: Bispectral index

BIC: Bicoherence index

EEG: Electroencephalography

QPC: Quadratic phase coupling

SFAR: French Society of Anesthesia and Reanimation

ASA: American Society of Anesthesiologists.

\section{Data Availability}

Data used in this work are from references and free databases mentioned in the text

\section{Conflicts of Interest}

The author declares that they have no conflicts of interest.

\section{References}

[1] E. Robert, "Epistemological problems," in Difficulty Naming and Defining Prion Diseases. Editions Harmattan, vol. 1, l'Harmattan, France, 2013, http://www.editions-harmattan.fr/index .asp?navig=catalogue\&obj=result.

[2] V. Billard, F. Servin, and S. Molliex, "Monitoring depth of general anesthesia," EMC Anesthesia \& Reanimation, vol. 2, no. 1, pp. 23-45, 2005.

[3] "Montaine Bernard," Methodology for Analyzing Neuronal Synchronizations in EEG Signals Using Time-Frequency Information Graphs, [PHD Thesis], Doctoral School: Engineering Sciences and Aeronautics, Research Sector: Signal and Image Processing, University of Poitiers, France, 2006.

[4] M. Caparos, "Automatic analysis of temporal lobe epileptic seizures from surface EEGs," Nancy Center for Automatic Research CNRS-UMR, vol. 7039, 2010.

[5] M. Billiard, Normal and Pathological Sleep: Sleep and Wake Disorders, Masson, Paris, 1994.

[6] B. Med, Hardware Architecture for Processing Physiological Signals in Real Time and Adaptable to the Variation of the Operating Environment, [PHD Thesis], National School of Engineering of sfax, Tunisia, 2009.

[7] Y. Tran and R. Din, Celestin Thierry and Aillet Morgan, Cerebral Functional Image, pp. 10-12, Docplayer, France, 2008, http://docplayer.fr/20382902-Imageries-fonctionnellescerebrales.html.

[8] F. Wendling, "Complexity of neural systems: contribution of signal processing and modeling in the interpretation of epileptic activities," in Signal and Image Processing Laboratory, INSERM U642, vol. 2, pp. 3-9, University of Rennes, 2002.

[9] R. R. Aguilar, J. A. Lopez, and I. O. Pineda, "Analysis of EEG signal processing techniques based on spectrograms," Research in Computing Science, vol. 145, no. 1, pp. 151-162, 2017.

[10] C. R. D. Oliveira, W. M. Bernardo, and V. M. Nunes, "Benefit of general anesthesia monitored by bispectral index compared with monitoring guided only by clinical parameters. System- atic review and meta-analysis," Brazillian Journal of Anesthesiology, vol. 67, no. 1, pp. 72-84, 2017.

[11] V. Billard, "Surveillance de la profondeur de l'anesthésie," in Conférences d'actualisation, pp. 17-32, Elsevier, Paris, 1997, editions SFAR.

[12] F. Bretherton, "Resonant interactions between waves. The case of discrete oscillations," Journal of Fluid Mechanics, vol. 20, pp. 401-410, 1964.

[13] A. Miller, J. W. Sleigh, J. Barnard, and D. A. Steyn-Ross, “Does bispectral analysis of the electroencephalogram add anything but complexity? ", British Journal of Anaesthesia, vol. 92, no. 1, pp. 8-13, 2004.

[14] M. R. Raghuveer and C. L. Nikias, "Bispectrum estimate: a parametric approach. Acoustics, speech and signal processing," IEEE Transactions on speech processing, vol. 33, no. 5, pp. 1213-1230, 1985.

[15] D. Longrois, M. Hirschi, E. Junke, and C. Mesteilman, "Monitoring of the depth of anesthesia," JEPU journal, vol. 145, pp. 65-73, 2002.

[16] Q. Liu, Y.-F. Chen, S.-Z. Fan, M. F. Abbod, and J.-S. Shieh, "EEG signals analysis using multiscale entropy for depth of anesthesia monitoring during surgery through artificial neural networks," Computational and mathematical methods in medicine, 2015.

[17] H. W. Shin, H. J. Kim, Y. K. Jang et al., "Monitoring of anesthetic depth and EEG band power using phase lag entropy during propofol anesthesia," BMC Anesthesiology, vol. 20, no. $49,2020$.

[18] B. G. Fahy and D. F. Chau, "The technology of processed electroencephalogram monitoring devices for assessment of depth of anesthesia," Anesthesia \& Analgesia, vol. 7, pp. 111-126, 2018.

[19] S. Inaba, M. Hashimoto, M. Takahashi, Y. Motomura, and A. Kochi, "Comparison of BIS and Ramsay score for evaluation of sedation with propofol in ICU Masui," The Japanese Journal of Anesthesiology, vol. 56, no. 1, pp. 57-60, 2007.

[20] C. Weatherburn, R. Endacott, P. Tynan, and M. Bailey, “The impact of bispectral index monitoring on sedation administration in mechanically ventilated patients," Anesthesia and Intensive Care, vol. 35, no. 2, pp. 204-208, 2007.

[21] D. Zhao, Y. Xu, W. He, T. Li, and Y. He, “A comparison of bispectral index and sedation-agitation scale in guiding sedation therapy: a randomized controlled study in patients undergoing short-term mechanical ventilation," Chinese Critical Care Medicine, vol. 23, no. 4, pp. 220-223, 2011. 